\title{
THE EFFECT OF CHANNEL ESTIMATION ERROR ON THE THROUGHPUT OF BROADCAST CHANNELS
}

\author{
Ali Vakili, Masoud Sharif, and Babak Hassibi \\ California Institute of Technology \\ Department of Electrical Engineering \\ Pasadena, CA 91125, USA
}

\begin{abstract}
In a broadcast channel in which one transmitter serves $n$ receivers, the capacity region highly depends on the amount of channel state information (CSI) at the transmitter. Assuming that the transmitter knows the SNR of all the receivers, opportunistic strategy maximizes the throughput (sum-rate) of the system. It is usually assumed that CSI is accurate, however, evaluating the SNR is basically an estimation problem in the receiver which cannot be done without error. In this paper, we analyze the effect of the noisy estimation of SNR on the throughput of a broadcast channel. We propose a generalization of the opportunistic transmission in which the transmitter still sends to the user with the highest estimated SNR, but backs off on the transmit rate based on the variance of the estimation error. We obtain the optimum amount of back off and compute the throughput for our scheduling scheme. Clearly, the estimation can be improved by using a longer training phase; however, longer training would deteriorate the throughput. In the final part of the paper, we address this trade off and obtain the optimum training strategy that maximizes the throughput of the system.
\end{abstract}

\section{INTRODUCTION}

A multiuser channel in which a transmitter sends independent streams of information to a number of receivers is commonly referred to as a Broadcast Channel (BC). In a timevarying environment such as a wireless channel, it is known that exploiting the multiuser nature of the channel by opportunistic transmission maximizes the throughput of such a system [1]. This however requires the full knowledge of the channel state information (CSI) at the transmitter and the receivers. From a practical perspective, obtaining CSI at the receivers is basically an estimation problem which in general cannot be error free. Therefore, it would be worth-

This work was supported in part by the National Science Foundation under grant no. CCR-0133818 and CCR-0326554, by the David and Lucille Packard Foundation, and by Caltech's Lee Center for Advanced Networking. while to understand the effect of channel estimation error on the capacity region and scheduling schemes in BC.

The effect of imperfect CSI on the capacity of point-topoint SISO and MIMO channels has been studied in [2], [8], and [3]. The robustness of the capacity achieving scheme in a Gaussian flat-fading channel is studied in [4]. As for the broadcast channel, [5] considered the effect of non-ideal feedback only in the transmitter due to doppler effect.

In this paper, we first take into account the effect of estimation noise and assume that the transmitter and receivers have an "estimate" of the channel and also know the variance of the estimation error. We further assume that the feedback link is error free. We consider a scheduling scheme that transmits to the user with the best estimated SNR at a rate which is backed off from the rate that would have been given by opportunistic scheduling. In our model we assume a packet is dropped if a capacity outage occurs. Backing off on the rate would therefore decrease the probability of occurrence of an outage. We obtain the optimum rate back off that maximizes the throughput. This optimum value can be found by solving a non-linear equation. In order to get more insight into the amount of back off as a function of the estimation noise, we obtain the back off explicitly for two regimes: (1) small variance of the estimation error and (2) large number of users. We then investigate the throughput loss due to this type of imperfect CSI. We obtain an approximation for the throughput in small error variance regime. Simulation results are presented to show the effect of channel estimation error on the throughput and also to verify our asymptotic analysis.

In our model in the first part, we assume that the channel estimations are given by a "genie" without any cost. In the second part of this paper, we consider a training phase in time at the beginning of each coherence interval of the channel [8]. Clearly, the variance of the estimation error is a function of the quality of the training which depends on its length and the amount of power spent during training. Increasing the throughput requires a better estimation for the SNR or a longer training period which itself results in sending fewer data symbols or less throughput. We analyze this 
trade off and show that the optimum training strategy which maximizes the throughput is to dedicate only one channel use for training in each coherence interval of the channel.

\section{THE CHANNEL MODEL}

We consider a single-antenna broadcast channel with $n$ users. We assume a block fading model for the channel with coherence interval of length $T$. Links to the users are assumed to be uncorrelated flat-fading Gaussian channels. We denote the signal sent to the $i$ 'th user in the $t$ 'th channel use by $S_{i}(t)$. Therefore the signal received by the $i$ 'th user can be written as,

$$
X_{i}(t)=\sqrt{\rho} h_{i}(t) S_{i}(t)+v_{i}(t), \quad i=1, \ldots, n,
$$

in which $h_{i}(t)$ is the channel coefficient with complex Gaussian distribution $\mathcal{C N}(0,1)$. We have normalized the signals so that $E\left\|S_{i}(t)\right\|^{2}=1$ and $\rho$ is the average signal to noise ratio of the received signal due to the channel noise alone. In $(1), v_{i}(t)$ is the additive white noise with i.i.d. $\mathcal{C N}(0,1)$ entries. Since we average the throughput over all channel uses, we drop the time index $t$ in (1).

We denote our estimation of the channel's coefficient and the estimation error by $\hat{h}_{i}$ and $\tilde{h}_{i}$, respectively. Thus,

$$
h_{i}=\hat{h}_{i}+\tilde{h}_{i}
$$

where $\hat{h}_{i}$ and $\tilde{h}_{i}$ have zero-mean complex Gaussian distributions. In fact $\rho\left|\hat{h}_{i}\right|^{2}$ is what the receiver $i$ would feedback to the transmitter as the estimated SNR. We further assume the feedback link is error free.

If we assume that the channel is estimated by an MMSE estimator, the orthogonality principle implies $E\left(\left|h_{i}\right|^{2}\right)=$ $E\left(\left|\hat{h}_{i}\right|^{2}\right)+E\left(\left|\tilde{h}_{i}\right|^{2}\right)[6]$. We further assume that the variance of the estimation error is equal to $\beta$, i.e., $E\left(\left|\tilde{h}_{i}\right|^{2}\right)=\beta$, and is known to the transmitter. The actual (instantaneous) SNR of the $i$ 'th user (denoted by $\lambda_{i}$ ) can be written as,

$$
\lambda_{i}=\frac{\left|\hat{h}_{i}\right|^{2}}{\frac{1}{\rho}+\left|\tilde{h}_{i}\right|^{2}}
$$

For mathematical convenience, we denote the normalized estimated SNR and the normalized estimation error for the $i$ 'th user by $\hat{\lambda}_{i}$ and $z_{i}$. Therefore, both $\hat{\lambda}_{i}$ and $z_{i}$ have exponential distribution of variance one and can be written as

$$
\hat{\lambda}_{i}=\frac{\left|\hat{h}_{i}\right|^{2}}{1-\beta}, \quad z_{i}=\frac{\left|\tilde{h}_{i}\right|^{2}}{\beta}
$$

We can now rewrite $\lambda_{i}$ in (3) as

$$
\lambda_{i}=\frac{\hat{\lambda}_{i}}{\frac{1}{\rho_{\text {eff }}}+\alpha z_{i}}
$$

where $\alpha=\frac{\beta}{1-\beta}$ and $\rho_{e f f}=(1-\beta) \rho$ denote "normalized error variance" and "effective channel SNR", respectively.

\section{THE OPPORTUNISTIC SCHEME WITH RATE BACK OFF}

In this paper, we assume that the transmitter knows $\alpha$ and $\rho_{\text {eff }}$ and the receivers feed back the estimated SNRs to the transmitter. We consider an opportunistic scheduling in which all the power is assigned to the user with the highest estimated SNR (referred to as the "best" user). Since the received SNR is just an estimate of the actual SNR, the transmitter backs off on the estimated SNR (or rate) in order to reduce the probability of outage. The main goal of this section is to compute the optimum amount of back off that maximizes the throughput (or sum-rate) as a function of the variance of the estimation error.

In order to find the throughput of this scheme we need to obtain the distribution of the actual SNR defined in (5) given the estimated SNR or $\hat{\lambda}_{i}$ for the best user. We drop the index $i$ whenever we denote the quantities related to the best user. The distribution of the maximum estimated SNR can be written as

$$
\operatorname{Pr}(\hat{\lambda})=n e^{-\hat{\lambda}}\left(1-e^{-\hat{\lambda}}\right)^{n-1} u(\hat{\lambda})
$$

where $u(\cdot)$ denotes the unit step function. We can further show that the distribution of the actual SNR of the best user given its estimation can be written as [7],

$$
\operatorname{Pr}(\lambda \mid \hat{\lambda})=e^{-\frac{1}{\alpha}\left(\frac{\hat{\lambda}}{\lambda}-\frac{1}{\rho_{e f f}}\right)} \frac{\hat{\lambda}}{\alpha \lambda^{2}} u\left(\frac{\hat{\lambda}}{\lambda}-\frac{1}{\rho_{e f f}}\right)
$$

If there was no error in our estimation, the actual SNR would have been $\rho \hat{\lambda}$. In fact, the actual SNR is always less than $\rho \hat{\lambda}$ in the presence of estimation error. In our scheduling, the transmitter backs off from $\rho \hat{\lambda}$ to $\lambda_{c}(\hat{\lambda})$ which is called the "assumed" SNR. Clearly, if the actual SNR (i.e., $\lambda$ ) is below the assumed SNR (i.e., $\lambda_{c}(\hat{\lambda})$ ), a capacity outage occurs. Therefore, the instantaneous rate will be,

$$
R= \begin{cases}\log \left(1+\lambda_{c}\right) & \lambda_{c} \leq \lambda \\ 0 & \lambda_{c}>\lambda\end{cases}
$$

Thus we can write the average throughput as,

$$
\begin{aligned}
R_{a v}= & \int_{0}^{\infty} n\left(1-e^{-\hat{\lambda}}\right)^{n-1} e^{-\hat{\lambda}} \log \left(1+\lambda_{c}(\hat{\lambda})\right) \\
& \times\left\{\int_{\lambda_{c}(\hat{\lambda})}^{\rho_{e f f} \hat{\lambda}} e^{-\frac{1}{\alpha}\left(\frac{\hat{\lambda}}{\lambda}-\frac{1}{\rho_{e f f}}\right)} \frac{\hat{\lambda}}{\alpha \lambda^{2}} d \lambda\right\} d \hat{\lambda}
\end{aligned}
$$

The following theorem obtains the optimum value for the back off to maximize the throughput of the system.

Theorem 1. Consider a broadcast channel with $n$ users where each user can estimate its channel with an error variance of $\beta$. The optimum backed off SNR (denoted by $\lambda_{c}^{o p t}$ ) 
that maximizes the throughput of the opportunistic scheduling is the non-trivial solution to the equation,

$$
\frac{\lambda_{c}^{o p t}}{\left(1+\lambda_{c}^{o p t}\right) \log \left(1+\lambda_{c}^{o p t}\right)}=\frac{1}{e^{\frac{1}{\alpha}\left(\frac{\hat{\lambda}}{\lambda_{c}^{o p t}}-\frac{1}{\rho_{e f f}}\right)}-1} \frac{\hat{\lambda}}{\alpha \lambda_{c}^{o p t}} .
$$

Proof: It is easy to see that the maximization can be interchanged with the integration. Maximization of the inner integral in (9) over $\lambda_{c}$ leads to (10).

When $\alpha=0$, Theorem 1 implies that $\lambda_{c}^{o p t}=\rho \hat{\lambda}$ which is consistent with the opportunistic scheduling in the absence of the estimation error.

Remark: It is worth mentioning that the actual SNR (defined in (3)) is always less than the estimated one, i.e., $\rho\left|\hat{h_{i}}\right|^{2}$. Therefore, for any nonzero $\beta$, if the transmitter sends at a rate of $\log (1+\rho \hat{\lambda})$, the packet will be dropped and the average throughput will become zero. This implies that a back off system is necessary in the presence of channel estimation error.

Although numerical evaluation of $\lambda_{c}$ is quite straightforward, finding an analytical solution for $\lambda_{c}^{o p t}$ does not seem to be tractable. Therefore we consider two important asymptotic regimes, namely, when the estimation noise $\beta$ is small and when the number of users $n$ is large.

\section{1. $\lambda_{c}^{o p t}$ for small- $\alpha$ regime}

From a practical point of view, we are mostly interested in the case where the estimation error (i.e., $\beta$ or equivalently $\alpha$ ) is small. Therefore in this subsection we fix $n$ and $\rho$ and let $\alpha$ go to zero. We can state the following theorem for this regime which is proved in [7],

Theorem 2. Consider the setting of Theorem 1. If $\alpha$ tends to zero, then $\lambda_{c}^{o p t}$ is equal to,

$$
\lambda_{c}^{o p t}=\frac{\rho_{e f f} \hat{\lambda}}{1+\alpha \rho_{e f f} \log \left(\frac{1}{\alpha}\right)+O(\alpha)} .
$$

Therefore we may write an approximation for $\lambda_{c}^{o p t}$ to the first order as,

$$
\lambda_{c}^{(s m a l l-\alpha, o p t)} \approx \frac{\rho_{e f f} \hat{\lambda}}{1+\alpha \rho_{e f f} \log \left(\frac{1}{\alpha}\right)}
$$

This can be used to find an approximation for the throughput of the system when $\alpha$ is small. In order to calculate $\mathrm{R}_{a v}$, one should plug in the solution of $\lambda_{c}^{o p t}$ into the integral of (9) and calculate the integral; this is however analytically intractable. Using Theorem 2 we can find an approximation for $R_{a v}$ when $\alpha$ is small by plugging (11) into (9) as

$$
\begin{aligned}
\mathrm{R}_{a v}^{\text {small }-\alpha} \approx & (1-\alpha) \int_{0}^{\infty} n\left(1-e^{-\hat{\lambda}}\right)^{n-1} e^{-\hat{\lambda}} \\
& \times \log \left[1+\frac{\rho_{\text {eff }} \hat{\lambda}}{1+\alpha \rho_{\text {eff }} \log \left(\frac{1}{\alpha}\right)}\right] d \hat{\lambda}
\end{aligned}
$$

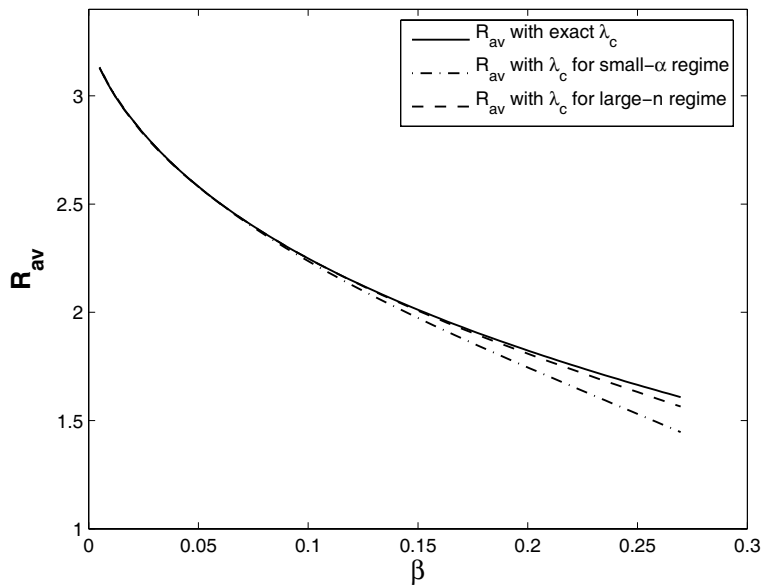

Fig. 1. Comparison of $R_{a v}$ calculated by using the exact value of $\lambda_{c}^{o p t}$ and small- $\alpha$ or large- $n$ approximations for $n=100$ and $\rho=5$

This essentially implies that

$\mathrm{R}_{a v}^{s m a l l-\alpha} \approx(1-\alpha) \mathrm{R}_{a v}^{n o-e r r}\left(\mathrm{SNR}=\frac{\rho_{\mathrm{eff}} \hat{\lambda}}{1+\alpha \rho_{\mathrm{eff}} \log \left(\frac{1}{\alpha}\right)}\right)$

where $\mathrm{R}_{a v}^{n o-e r r}(x)$ is the sum-rate capacity in a broadcast channel with no estimation error and with average SNR of $x$. Therefore when $\beta$ is small, the estimation error has two effects on the throughput namely, a pre-log factor of $(1-\alpha)$ and also the SNR hit of $\frac{1}{1+\alpha \rho_{\text {eff }} \log \left(\frac{1}{\alpha}\right)}$.

\section{2. $\lambda_{c}^{o p t}$ for large- $n$ regime}

In a typical cellular system, we may have a large number of users $n$. This implies that, almost surely $\hat{\lambda}$ is about $\log n \pm$ $\log \log n$. In this regime, we can obtain $\lambda_{c}^{o p t}$ and prove that [7],

$$
\lambda_{c}^{o p t}=\frac{\rho_{e f f} \hat{\lambda}}{1+\alpha \rho_{e f f} \log \log \frac{\hat{\lambda}}{\alpha}+O(\log \log \log n)} .
$$

Fig. 1 shows the numerical results for average throughput when $\lambda_{c}^{o p t}$ is computed numerically by solving (10), using small- $\alpha$ approximation, and using large- $n$ approximation. Here we assume that there are $n=100$ users in the system and $\rho=5$. Fig. 2 shows the throughput as a function of the number of users for different $\beta$ 's. It is worth noting that by only little estimation error of $\beta=0.05$, the throughput is decreased by about $\% 20$.

\section{ESTIMATION VIA TRAINING IN TIME}

In the previous section, we assumed that the channel estimation is given to the system with the aid of a "genie" at no cost. In this section, we take into account the benefits and 
costs of a training phase in the system. Following [8], we assume that at the beginning of each coherence interval of length $T^{1}$, there is a training phase of duration $T_{\tau}$ and data will be transmitted in the remaining part, i.e., $T_{d}=T-T_{\tau}$. We also assume that we have an energy budget equal to $E$ for each coherence interval. If we define $\rho_{\tau}$ and $\rho_{d}$ as the power levels of the training and data phases respectively, this implies that,

$$
E=\rho_{\tau} T_{\tau}+\rho_{d} T_{d}
$$

It is intuitively clear that as we increase the training budget (i.e., $T_{\tau}$ or $\rho_{\tau}$ ), the estimation error will decrease and therefore the transmission rate during the data phase $T_{d}$ will be increased. This however would decrease the overall throughput as the duration of data transmission (or its power) will diminish. Therefore there is a trade off on the amount of training needed to maximize throughput.

Considering an MMSE estimation, we can show that the variance of the estimation error is [6],

$$
\beta=\frac{1}{1+\rho_{\tau} T_{\tau}}=\frac{1}{1+E_{\tau}} .
$$

The next theorem states the optimum training power and duration that maximize the throughput.

Theorem 3. Consider the settings of Theorem 1 and assume that the channel estimation is obtained by MMSE training phase of length $T_{\tau}$ with power level of $\rho_{\tau}$. In order to maximize the throughput, we need only one symbol, i.e. $T_{\tau}=1$, for training. The optimum power level $\rho_{\tau}$ is the solution to a maximization problem that can be found numerically.

Proof: The proof follows the method used in [8]. We find the derivative of the expression for $R_{a v}$ with respect to $T_{d}$ and show that it is positive. Since $T_{\tau}$ can not be less than 1 , we should fix it at this value. See [7] for the complete proof and the formulation of the maximization problem.

The result of Theorem 3 implies that using more than one channel use for training would deteriorate the throughput although it does improve the channel estimation. This is mainly due to the fact that using a longer training decreases the pre-log factor while improves the signal to noise ratio in the argument of the logarithm.

\section{CONCLUSION}

We considered a $\mathrm{BC}$ with receivers having channel estimation error. We generalized the opportunistic scheme to a transmission strategy where we send to the user with the highest estimated SNR with a rate back off. We obtained the optimum rate back off and the resulting throughput. We

$$
\text { ber. }
$$

${ }^{1} T$ denotes the number of channel uses and therefore is a discrete num-

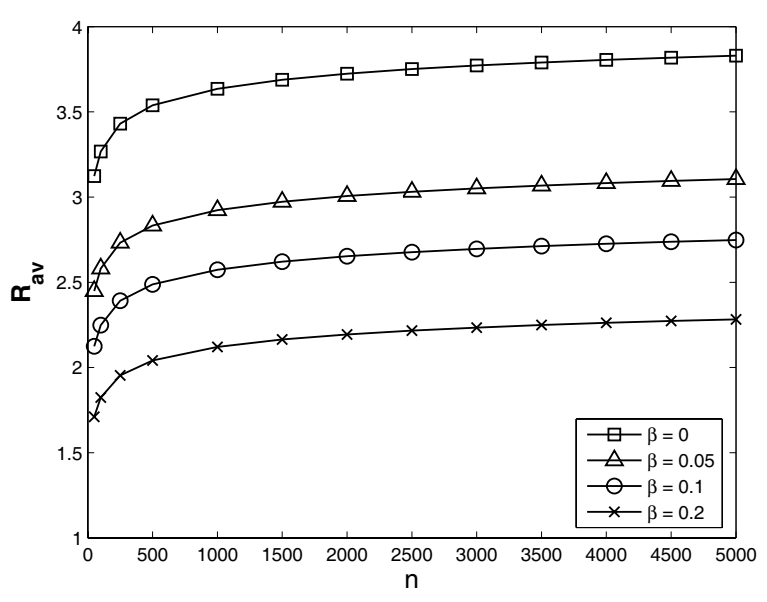

Fig. 2. The effect of the estimation error on the throughput for $\rho=5$

further analyzed the behavior of the back off when the variance of the error is small or when the number of users is large. We also looked into the trade offs when we have a training phase on the throughput. We showed that in order to maximize the throughput, we need to spend only one channel use per coherence interval for training.

\section{REFERENCES}

[1] R. Knopp and P. Humblet, "Information capacity and power control in single cell multiuser communications," in Proc. IEEE Inter. Conf. Comm., pp. 331- 335, 1995.

[2] M. Medard, "The effect upon channel capacity in wireless communications of perfect and imperfect knowledge of the channel," IEEE Trans. on Info. Theory, vol. 46, no. 3, pp. 933-946, May 2000.

[3] T. Yoo and A. Goldsmith, "Capacity and optimal power allocation for fading MIMO channels with channel estimation error," submitted to IEEE Trans. on Info. Theory, Aug. 2005.

[4] A. Lapidoth and S. Shamai, "Fading channels: How perfect need "perfect side information" be?," IEEE Trans. on Info. Theory, vol. 49, no. 10, pp. 951-964, April 2002.

[5] M. Kobayashi, G. Caire, and D. Gesbert, "Antenna diversity vs. multiuser diversity: Quantifying the tradeoffs," in Proc. ISITA, October, 2004.

[6] T. Kailath, A.H. Sayed, and B. Hassibi, Linear Estimation, Prentice-Hall, 2000.

[7] A. Vakili, M. Sharif, and B. Hassibi, "The effect of SNR estimation error on the sum-rate of broadcast channels," in preparation.

[8] B. Hassibi and B. M. Hochwald, "How much training is needed in a multiple-antenna wireless link?," IEEE Trans. on Info. Theory, vol. 48, no. 5, pp. 1118-1134, May 2003. 